\title{
Ultrafast Interfacial Carrier Dynamics and Persistent Topological Surface States in VSe2/Bi2Se3 Van Der Waals Heterojunctions
}

\section{Tae Gwan Park}

Korea Advanced Institute of Science and Technology (KAIST)

Jae Ho Jeon

Sejong University

Seung-Hyun Chun

Sejong University https://orcid.org/0000-0001-8397-4481

\section{Sunghun Lee}

Sejong University https://orcid.org/0000-0002-1974-8110

Fabian Rotermund ( $\nabla$ rotermund@kaist.ac.kr)

Korea Advanced Institute of Science and Technology (KAIST)

\section{Article}

Keywords: vanadium diselenide (VSe2), bismuth selenide (Bi2Se3), van der Waals heterostructures, ultrafast spectroscopy, interfacial phonon, carrier dynamics

Posted Date: January 19th, 2022

DOI: https://doi.org/10.21203/rs.3.rs-1206940/v1

License: (c) (i) This work is licensed under a Creative Commons Attribution 4.0 International License. Read Full License

Version of Record: A version of this preprint was published at Communications Physics on July 14th, 2022. See the published version at https://doi.org/10.1038/s42005-022-00961-9. 


\section{Ultrafast interfacial carrier dynamics and persistent}

\section{topological surface states in $\mathrm{VSe}_{2} / \mathrm{Bi}_{2} \mathrm{Se}_{3}$ van der}

\section{Waals heterojunctions}

Tae Gwan Park ${ }^{1}$, Jae Ho Jeon ${ }^{2}$, Seung-Hyun Chun ${ }^{2}$, Sunghun Lee ${ }^{2, *}$, Fabian Rotermund ${ }^{1, *}$

${ }^{1}$ Department of Physics, Korea Advanced Institute of Science and Technology (KAIST),

Daejeon 34141, Republic of Korea

${ }^{2}$ Department of Physics and Astronomy, Sejong University, Seoul 05006, Korea 


\section{ABSTRACT}

Vanadium diselenide ( $\mathrm{VSe}_{2}$ ) has recently been highlighted as an efficient 2D electrode owing to its extra-high conductivity, thickness-controllability, and van der Waals epitaxial contact. However, as the electrode, applications of $\mathrm{VSe}_{2}$ to various material systems are still lacking. Here, by employing ultrafast time-resolved spectroscopy, we study $\mathrm{VSe}_{2}$-thickness-dependent interfacial effects in heterostructures with topological insulator $\mathrm{Bi}_{2} \mathrm{Se}_{3}$ that is severely affected by contact with conventional 3D electrodes. Our results particularily show unaltered Dirac surface state of $\mathrm{Bi}_{2} \mathrm{Se}_{3}$ against forming junctions with $\mathrm{VSe}_{2}$, efficient ultrafast hot electron transfer from $\mathrm{VSe}_{2}$ to $\mathrm{Bi}_{2} \mathrm{Se}_{3}$ across the interface, significantly shortened metastable carrier lifetimes in $\mathrm{Bi}_{2} \mathrm{Se}_{3}$ due to dipole interactions enabling efficient current flow, and the electronic level shift ( $\sim$ tens meV) of bulk states of $\mathrm{Bi}_{2} \mathrm{Se}_{3}$ caused by interfacial interactions, which is $\sim 10$ times lower compared to conventional 3D electrodes, implying weak Fermi level pinning. Our observations confirm $\mathrm{VSe}_{2}$ as an ideal electrode for efficient $\mathrm{Bi}_{2} \mathrm{Se}_{3}$-based-applications with full utilization of topological insulator characteristics.

\section{KEYWORDS}

vanadium diselenide $\left(\mathrm{VSe}_{2}\right)$, bismuth selenide $\left(\mathrm{Bi}_{2} \mathrm{Se}_{3}\right)$, van der Waals heterostructures, ultrafast spectroscopy, interfacial phonon, carrier dynamics 


\section{INTRODUCTION}

Topological insulators (TIs) are a class of electronic materials that possess ordinary insulating states in the bulk and topologically protected metallic states at the surface. ${ }^{1,2}$ The topological surface state with massless Dirac fermions exhibits unique properties of spin-momentum locking ensured by $Z_{2}$ invariant in three-dimensional (3D) TIs including $\mathrm{Bi}_{2} \mathrm{Se}_{3}$. A spin-polarized and defect-tolerant conducting channel of these TIs enables promising electronic, optoelectronic, and spintronic applications, ${ }^{2,3}$ such as ultralow-power tunnel transistor, ${ }^{4,5}$ opto-spintronics with polarized photocurrent, ${ }^{6,7}$ high-performance broadband photodetector, ${ }^{8,9}$ and nonvolatile memory by spin-transfer torque. ${ }^{10}$ For device applications in transistors or external circuits, contact with metal electrodes plays a crucial role. However, in the junction with metal electrodes for producing transistors or external circuits, unexpected contact issues occur for several reasons such as interfacial hybridization and defects during the manufacturing process, leading to relaxation of spin-momentum locking and large contact resistance by strong Fermi level pinning (FLP). ${ }^{11,12}$ This can severely degrade the device performance. Moreover, an inherent low-dimensionality and the existence of Dirac surface states make the material systems themselves very sensitive to the junction interfaces. Although several conventional 3D metals, ${ }^{13-16}$ including $\mathrm{Au}, \mathrm{Cr}$, $\mathrm{Ti}$, and $\mathrm{Pd}$, have been adopted in $\mathrm{Bi}_{2} \mathrm{Se}_{3}$-based applications, the interfacial study of $\mathrm{Bi}_{2} \mathrm{Se}_{3}$-based junction has shown that there exist still several contact issues to be solved, such as relaxation of Dirac surface states and strong FLP due to chemical/electrical interaction (hybridization) at the interface except for $\mathrm{Au}$, which exhibits weak interaction with $\mathrm{Bi}_{2} \mathrm{Se}_{3}{ }^{12}$

A similar issue for the metal electrode contact has appeared at 2D semiconducting transition metal dichalcogenides (s-TMDs). ${ }^{17-20}$ The s-TMDs as the topological insulator are critically affected by such interactions at the interface with metal electrodes owing to their inherent 
atomically thin thickness and low dimensionality. ${ }^{18,19}$ As a breakthrough strategy, van der Waals (vdW) contact has been illuminated to form atomically sharp interfaces and suppress chemical interactions at interfaces between two dissimilar materials. ${ }^{18,20-23}$ The vdW metal-semiconductor junctions (MSJs) have been recently demonstrated as the ideal junction interfaces by transferring graphene $^{22,24-26}$ or noble 3D metal films. ${ }^{18,20-21}$ The desire for realizing an ideal junction interface and optimized metal electrode materials leads to considerable interest in metallic TMDs (mTMDs) beyond graphene..$^{22,27-30}$ The m-TMDs have been successfully adopted as metal electrodes for s-TMD, because they provide weak FLP in $\mathrm{vdW}$ MSJs, ${ }^{22}$ high field-effect mobility in $\mathrm{VTe}_{2} / \mathrm{MoS}_{2},{ }^{29}$ low contact resistance in $\mathrm{VS}_{2} / \mathrm{MoS}_{2}{ }^{30}$ with high functionality, an integrated external circuit with advantages of high-conductivity, tunable thickness, and van der Waals epitaxial contacts, ${ }^{27}$ in contrast to the use of conventional 3D metals. Among m-TMDs, $\mathrm{VSe}_{2}$, which exhibits thickness controllability and extra-high $\left(10^{2}-10^{4}\right.$ order $)$ electrical conductivity compared to other 2D materials, turned out to be a promising electrode material. ${ }^{28}$ Recently, $\mathrm{VSe}_{2}$ at metallic 1T-phase has been applied as the electrode in diverse 2D material systems such as $\mathrm{VSe}_{2} / \mathrm{WSe}_{2}$, $\mathrm{VSe}_{2} / \mathrm{MoSe}_{2} \mathrm{vdW}$ MSJs with low contact resistance, ${ }^{31}$ efficient electrocatalyst for Li-S batteries, ${ }^{32}$ and superior K-ion storage. ${ }^{33}$ Thus, it is considered important to expand and elucidate the role of $\mathrm{VSe}_{2}$ as the metal electrode in diverse material systems where the contact issue still remains.

In this work, we investigate the interfacial junction in $\mathrm{VSe}_{2} / \mathrm{Bi}_{2} \mathrm{Se}_{3}$ heterostructures (VBHs) by employing ultrafast optical spectroscopy in reflection geometry. This allows us to examine the interfacial phononic/carrier dynamics in a highly sensitive manner. ${ }^{34-37}$ Since the photoexcited carriers in $\mathrm{Bi}_{2} \mathrm{Se}_{3}$ are quite sensitive to the topological state ${ }^{38-40}$ and Fermi level, ${ }^{41-42}$ crucial information on certain modulation of both Dirac surface state and insulating bulk state of $\mathrm{Bi}_{2} \mathrm{Se}_{3}$ by junctions with metallic $\mathrm{VSe}_{2}$ can be obtained. An electron microscope and interfacial acoustic 
phonon dynamics show a good quality of interfacial contact in the VBHs. The hot electron transfer from $\mathrm{VSe}_{2}$ to $\mathrm{Bi}_{2} \mathrm{Se}_{3}$ across the vdW gap in ultrashort timescale ( $\left.100 \mathrm{fs}\right)$ leads to the improved efficiency of $\mathrm{Bi}_{2} \mathrm{Se}_{3}$-based optoelectronic devices. In addition, the photoexcited carrier dynamics in $\mathrm{VBH}$ provide direct evidence of unaltered surface Dirac state of $\mathrm{Bi}_{2} \mathrm{Se}_{3}$ by the contact with $\mathrm{VSe}_{2}$. The $\mathrm{VSe}_{2}$ thickness-dependent spectral and dynamical response in the VBHs quantitatively gives electronic modulation of the bulk band in $\mathrm{Bi}_{2} \mathrm{Se}_{3}$, which originated by interfacial dipole interactions due to the charge transfer/redistribution for thermal equilibrium. The observed electronic level shifts of $\mathrm{Bi}_{2} \mathrm{Se}_{3}$ by the junction with $\mathrm{VSe}_{2}$ are $\sim 10$ times lower compared to the junction with conventional 3D metals, indicating weak FLP. The lifetime of metastable carriers in $\mathrm{Bi}_{2} \mathrm{Se}_{3}$ is shortened by 3 times due to dipole interactions in $\mathrm{VBH}$, enabling highly efficient current flow. Overall results suggest that $\mathrm{VSe}_{2}$ can be considered as a promising candidate for the vdW metal electrode in $\mathrm{Bi}_{2} \mathrm{Se}_{3}$-based diverse applications by fully utilizing the superior characteristics of topological insulators.

\section{RESULTS AND DISCUSSION}

\section{Preparation and characterization of VBHs}

For the investigation, single-crystalline metallic $1 \mathrm{~T}-\mathrm{VSe}_{2}$ was grown by a chemical vapor transport method and transferred onto a 10 -quintuple layer $(\mathrm{QL}) \mathrm{Bi}_{2} \mathrm{Se}_{3}$ thin film by mechanical exfoliation. The carrier concentration $\left(N_{0}\right)$ of $\mathrm{Bi}_{2} \mathrm{Se}_{3}$ was approximately $N_{0}=3.13 \times 10^{20} \mathrm{~cm}^{-3}$ based on the Hall resistivity measurements (Fig. S1). The hall resistance of $10-\mathrm{QL} \mathrm{Bi}_{2} \mathrm{Se}_{3}$ shows the characteristics of an $n$-type $\mathrm{Bi}_{2} \mathrm{Se}_{3}$. As marked positions by black arrows in Fig. 1a, we were able to achieve different thicknesses of $\mathrm{VSe}_{2}$ on $\mathrm{Bi}_{2} \mathrm{Se}_{3}$ in a single flake. The size of a $\mathrm{VSe}_{2}$ flake on $\mathrm{Bi}_{2} \mathrm{Se}_{3}$ was sufficiently large compared to the spatial resolution $(<2 \mu \mathrm{m})$ of our spectroscopic system, and 
imperfectly exfoliated $\mathrm{VSe}_{2}$ flakes showed regions with different thicknesses, enabling us to investigate the $\mathrm{VSe}_{2}$ thickness-dependent phononic and carrier dynamics of $\mathrm{Bi}_{2} \mathrm{Se}_{3}$. Different positions of $\mathrm{VSe}_{2}$ with different thicknesses were marked as Pos. 1 to 4 , as shown in Fig. 1a (additionally see Figs. 1d,e). The conspicuous and clear Raman modes (Fig. 1b) and high-angle annular dark-field scanning transmission electron microscopy (HAADF-STEM) image (Fig. 1c) confirmed that both $\mathrm{VSe}_{2}$ and $\mathrm{Bi}_{2} \mathrm{Se}_{3}$ had a high-quality single-crystalline nature. Note that Figure $1 \mathrm{~b}$ shows the Raman spectra measured in individual $\mathrm{VSe}_{2}$ and $\mathrm{Bi}_{2} \mathrm{Se}_{3}$, since only the predominant $\mathrm{VSe}_{2}$ Raman peak at $206 \mathrm{~cm}^{-1}$ was obtained from the $\mathrm{VSe}_{2} / \mathrm{Bi}_{2} \mathrm{Se}_{3}$ heterostructures (VBHs) owing to relatively thick $\mathrm{VSe}_{2}$, which obscures the weak Raman signal of $\mathrm{Bi}_{2} \mathrm{Se}_{3}$ at appropriate laser powers below the $\mathrm{VSe}_{2}$ damage threshold. Each Raman mode showed in good agreement with the previous studies of $1 \mathrm{~T}-\mathrm{VSe}_{2}$ and $\mathrm{Bi}_{2} \mathrm{Se}_{3} \cdot{ }^{[43,44]}$ Note that the absence of Raman mode of the semiconducting $2 \mathrm{H}-\mathrm{VSe}_{2}$ at $190 \mathrm{~cm}^{-1},{ }^{[45]}$ which can be formed by structural phase transition during the preparation process, implies that the $\mathrm{VSe}_{2}$ used in this study was a pure metallic 1T-phase. The thicknesses of $\mathrm{VSe}_{2}$ at the marked positions $(1,2,3$, and 4) were measured to be $48,74,118$, and $167 \mathrm{~nm}$ on $10 \mathrm{QL} \mathrm{Bi}_{2} \mathrm{Se}_{3}$ by atomic force microscopy (AFM), respectively (Fig. 1e). The root means square roughness extracted from the AFM images (Fig. 1d) was quite low (under $\sim 0.1 \mathrm{~nm}$ ), indicating a clean and smooth surface.

\section{Interfacial coherent acoustic phonon (CAP) dynamics in VBHs}

To verify superior interfacial contact between $\mathrm{VSe}_{2}$ and $\mathrm{Bi}_{2} \mathrm{Se}_{3}$ in a large area, collinearly aligned two-colour pump-probe spectroscopy was carried out on VBHs. The pump (1.5 eV) and probe (1.0 $\mathrm{eV}$ ) pulses were normally incidents on the VBHs with a time delay $\left(\tau_{\mathrm{d}}\right)$. Time-resolved reflectance measurements were performed at each position of the VBHs. Inset of Fig. 2a illustrates a schematic 
of the process, where coherent acoustic phonons (CAPs) occurred at the interfaces of VBHs. The acoustic wave was generated at the $\mathrm{VBH}$ interfaces by the incident optical pulse and propagated into the $\mathrm{VSe}_{2}$ flake. Figure 2 shows the background-free CAPs in $10 \mathrm{QL} \mathrm{Bi} \mathrm{Se}_{3}$ and $118 \mathrm{~nm}$ $\mathrm{VSe}_{2} / 10 \mathrm{QL} \mathrm{Bi}_{2} \mathrm{Se}_{3}(118 \mathrm{~nm} \mathrm{VBH})$ obtained by abstraction from the fitting with a biexponential representing the decay dynamics (more details in Note S2 and Fig. S2). Earlier CAP before 40 ps and later echo signals around 80 ps time delay were observed. For the earlier CAP part, the oscillation frequencies of CAPs were obtained by fitting with damped oscillation functions as 125 and $43 \mathrm{GHz}$. The obtained frequencies well matched the interlayer vibrational modes in 10-QL $\mathrm{Bi}_{2} \mathrm{Se}_{3}$, in which the frequency depended on the number of layers. ${ }^{[4,46]}$ Note that the CAP signal of $\mathrm{Bi}_{2} \mathrm{Se}_{3}$ was observed in both $\mathrm{Bi}_{2} \mathrm{Se}_{3}$ and the $\mathrm{VBH}$ at the same time delay. This result reflected that $\mathrm{Bi}_{2} \mathrm{Se}_{3}$ was almost directly excited by the optical pulse owing to the sufficiently long penetration depth of $\mathrm{VSe}_{2}$ compared to $\mathrm{Bi}_{2} \mathrm{Se}_{3} .{ }^{[47,48]}$

After damping of the inherent earlier CAP signal in $\mathrm{Bi}_{2} \mathrm{Se}_{3}$, subsequent echo signals were observed. The acoustic wave generated by the optical pump reciprocated in $\mathrm{VSe}_{2}$ by reflection at the air and $\mathrm{Bi}_{2} \mathrm{Se}_{3}$ interface as shown in the inset of Fig. 2a. When the acoustic wave arrived at the $\mathrm{VSe}_{2}-\mathrm{Bi}_{2} \mathrm{Se}_{3}$ interface, echo signals appeared with different intervals depending on the thickness of $\mathrm{VSe}_{2}$ (Fig. 2b). The acoustic waves at interfaces can be described by the acoustic mismatch (AM) model for boundary conditions of continuous displacement and stress. This indicates perfect and atomically flat interfaces in our case. ${ }^{[4]}$ Based on AM model, the amplitude reflection coefficient is given by

$$
r_{i j}=\frac{Z_{j}-Z_{i}}{Z_{j}+Z_{i}}
$$

Here, $Z=\rho s$ is the acoustic impedance of material, where $\rho$ and $s$ are the density and the sound speed, respectively. The amplitude reflection coefficients of interfacial CAP were estimated 
to be $r_{V S e_{2}-a i r} \approx-1$ and $r_{V S e_{2}-B i_{2} S e_{3}}=0.29$ with $Z_{B i_{2} S e_{3}}=21.78,{ }^{[50]} Z_{V S e_{2}}=12$, and $Z_{\text {air }}=0.0004$ with a unit of $10^{6} \mathrm{~kg} \mathrm{~m}^{-2} \mathrm{~s}^{-1}$. The negative reflection coefficient indicates that the phase of the reflected acoustic wave is changed by $\pi$. Hence, the reflected echo exhibits a phase shift of $\pi$ with each round trip, resulting from the reflection at the air interfaces. In the case of the heterostructure of $118 \mathrm{~nm} \mathrm{VSe} 2$ and 10-QL $\mathrm{Bi}_{2} \mathrm{Se}_{3}(118 \mathrm{~nm} \mathrm{VBH}$ in Fig. 2a), the first echo was observed at $87 \mathrm{ps}$, and subsequently, the second and third echoes emerged with alternating phases at the same interval of $86 \mathrm{ps}$. The similar times of the first echo (87 ps) and the interval (86 ps) imply that acoustic waves were launched from $\mathrm{Bi}_{2} \mathrm{Se}_{3}$ through hot phonons and interlayer vibration after photoexcitation. Note that the reflected acoustic waves at the interface of $\mathrm{Bi}_{2} \mathrm{Se}_{3} / \mathrm{Al}_{2} \mathrm{O}_{3}$ substrate were negligible in our analysis because the reflection coefficient of $\mathrm{Bi}_{2} \mathrm{Se}_{3} / \mathrm{Al}_{2} \mathrm{O}_{3}$ is 0.18 with the acoustic impedance for $\mathrm{Z}_{\mathrm{Al}_{2} \mathrm{O}_{3}}=31.5 \times 10^{6} \mathrm{kgm}^{-2} \mathrm{~s}^{-1[51]}$ does not change the phase of acoustic waves. Moreover, the expected echo interval in $\mathrm{Bi}_{2} \mathrm{Se}_{3}$ at $3.4 \mathrm{ps}$, where the thickness of $\mathrm{Bi}_{2} \mathrm{Se}_{3}$ is $10 \mathrm{~nm}$ and the sound speed is $2.9 \mathrm{~nm} / \mathrm{ps}$, is substantially short compared to that observed in $\mathrm{VSe}_{2}$. The phase was also not changed by reflection at $\mathrm{Bi}_{2} \mathrm{Se}_{3} / \mathrm{Al}_{2} \mathrm{O}_{3}$ interfaces, thus, we safely concluded that the echo signals were observed when the acoustic waves reached the interface at $\mathrm{VSe}_{2} / \mathrm{Bi}_{2} \mathrm{Se}_{3}$.

The observed time intervals between echoes of $42,54,86$, and $111 \mathrm{ps}$ in the $48,74,118$, and $167 \mathrm{~nm}$ VBHs, respectively (Fig. 2b), exhibited a linear dependence on the $\mathrm{VSe}_{2}$ thickness, which means that so did the transit round-trip time of the acoustic wave in $\mathrm{VSe}_{2}$. The inset of Figure $2 \mathrm{~b}$ shows the $\mathrm{VSe}_{2}$-thickness-dependent time interval between echoes at each $\mathrm{VBH}$, with a sound velocity of $\mathrm{VSe}_{2}$ of $v_{\mathrm{s}}=2.71 \mathrm{~km} / \mathrm{s}$ estimated from the linear dependence. This value is close to the previously reported value of $2.67 \mathrm{~km} / \mathrm{s} \cdot{ }^{[52]}$ In general, defects and imperfect contact can reduce the coherence of acoustic waves and induce different frequencies, which is independent of 
the material thickness. ${ }^{[49,53]}$ Moreover, as shown in Fig. 2e, the different amplitudes between echoes were consistent with the reflection coefficient of $r_{V S e_{2}-B i_{2} S e_{3}}=0.29$, while $r$ vSe2-air was near unity, providing direct evidence of an atomically flat interface with negligible defects or poor contact of the VBHs. ${ }^{[54]}$ Consequently, the interface of each VBH was assumed to be nearly perfect, and the effects of additional gap states due to defects that hinder the interpretation of the results could be excluded.

\section{Ultrafast hot electron dynamics of Dirac surface states at VBHs}

With the VBHs possessing a high-quality interface, we performed transient reflectance (TR) measurements to investigate the interfacial carrier dynamics in the metallic surface and the insulating bulk electronic states of $\mathrm{Bi}_{2} \mathrm{Se}_{3}$ at junctions with $\mathrm{VSe}_{2}$. In TR measurements with a pump fluence of $12 \mu \mathrm{J} / \mathrm{cm}^{2}$, the photoexcited carrier density estimated from the power density along with the complex refractive index of $\mathrm{Bi}_{2} \mathrm{Se}_{3}{ }^{[48]}$ was $1.6 \times 10^{19} \mathrm{~cm}^{-3}$, which is only $5 \%$ of the background carrier concentration and implies a small perturbation to the charge carriers in $\mathrm{Bi}_{2} \mathrm{Se}_{3}$. Figure $3 \mathrm{a}$ shows TR spectra for $10 \mathrm{QL} \mathrm{Bi}_{2} \mathrm{Se}_{3}$, bare $\mathrm{VSe}_{2}$, and $118 \mathrm{~nm} \mathrm{VBH}$. For bare $\mathrm{Bi}_{2} \mathrm{Se}_{3}$, the TR spectral responses were significantly different before and after $\sim 2$ ps. Just after photoexcitation, the negative differential reflectance $\left(\Delta R / R_{0}\right)$ at overall probe energies was observed before $2 \mathrm{ps}$, whereas, after $2 \mathrm{ps}$, a derivative-like TR response, i.e. changing the TR sign from negative to positive according to probe energy, was observed. Since the observed carrier dynamics are complex due to the coexistence of the metallic surface state and insulating bulk state of $\mathrm{Bi}_{2} \mathrm{Se}_{3}$ thin

film in optical spectroscopy ${ }^{[39,55]}$ a clear distinction is necessary for each contribution. The overall negative $\Delta \mathrm{R} / \mathrm{R}_{0}$ before 2 ps was confirmed by the transient absorption (TA) measurement results (Fig. S3) to be related to the photobleaching through the injection of hot carriers ${ }^{[55,56]}$ as indicated 
by stage (i) in Figs 3b,c. After hot carrier injection, the rapid decay with $2.5 \mathrm{ps,}$ as indicated $\tau_{1}$ and stage (ii), was attributed to the phonon-mediated bulk-surface scattering of excited carriers in $\mathrm{Bi}_{2} \mathrm{Se}_{3},{ }^{[39,55,56]}$ which implies a dominant contribution of the Dirac surface state on carrier dynamics in $\mathrm{Bi}_{2} \mathrm{Se}_{3}$. After surface scattering relaxation, the derivative-like TR spectra (positive $\Delta \mathrm{R} / \mathrm{R}_{0}$ to negative $\Delta \mathrm{R} / \mathrm{R}_{0}$ according to probe energy) with a long lifetime appeared after $\sim 5 \mathrm{ps}$. The derivative TR with long decay time was similar to the previously reported relaxation time of bulk state toward their respective bottom bands, i.e. conduction band minimum/valence band maximum (CBM/VBM) for the bulk state. ${ }^{[55]}$ The derivative-like TR spectra and their decay dynamics, which are commonly observed in semiconductors by excitonic effects or state filling by photoexcitation, ${ }^{[34,36,37,57]}$ can be regarded as a signature of the insulating bulk band of $\mathrm{Bi}_{2} \mathrm{Se}_{3}$ as discussed in the following section.

For bare $\mathrm{VSe}_{2}$ on $\mathrm{SiO}_{2}$ substrate with a thickness of about $100 \mathrm{~nm}$ (Fig. S4a), the TR spectra show a negative $\Delta \mathrm{R} / \mathrm{R}_{0}$ in overall delay time and probe energy. Owing to the metallic electronic band structures with an energy difference of about $1.7 \mathrm{eV}$ between Fermi energy and valence band maximum, ${ }^{[58]}$ the photobleaching of intraband transition could occur in our spectral range on the order of $1 \mathrm{eV}$. The excited electron dynamics of $\mathrm{VSe}_{2}$ were considerably slow compared to $\mathrm{Bi}_{2} \mathrm{Se}_{3}$ as shown in Fig. 3b. The excited electrons in $\mathrm{VSe}_{2}$ did not recover at all within a given time window (only 10-25\% even within 300 ps time delay, as shown in Fig. S4b). The relaxation time $\left(\tau_{\text {relax }}\right)$ of hot electrons in $\mathrm{VSe}_{2}$ is about $1.5 \mathrm{ps}$, which is similar to the monolayer limit ${ }^{[59]}$, but the $\mathrm{TR}$ response of $\mathrm{VSe}_{2}$ films including the sign of $\Delta \mathrm{R} / \mathrm{R}_{0}$ and decay kinetics significantly differs from the monolayer $\mathrm{VSe}_{2},{ }^{[59]}$ which indicates that the excited electron dynamics have a thickness dependence as for other TMD systems. ${ }^{[60,61]}$ Interestingly, for $118 \mathrm{~nm}$ $\mathrm{VBH}$, the TR response and kinetics are almost the same as $\mathrm{Bi}_{2} \mathrm{Se}_{3}$. As shown in Fig. 3b, the decay 
characteristics $\left(\tau_{1}\right)$ of the surface state scattering indicate that the role of the Dirac surface state in $\mathrm{Bi}_{2} \mathrm{Se}_{3}$ was well preserved at the interfacial junction with $\mathrm{VSe}_{2}$. Furthermore, it is noteworthy that the positive $\Delta \mathrm{R} / \mathrm{R}_{0}$ at $\mathrm{VBH}$ is improved by $\sim 2$ times compared to $\mathrm{Bi}_{2} \mathrm{Se}_{3}$ without $\mathrm{VSe} \mathrm{e}_{2}$, although the $\Delta \mathrm{R} / \mathrm{R}_{0}$ for bare $\mathrm{VSe}_{2}$ films is negative. The expected hot electron transfer time ( $\left.\tau_{\text {trans }}\right)$ from $\mathrm{VSe}_{2}$ to $\mathrm{Bi}_{2} \mathrm{Se}_{3}$ is comparable or faster than $100 \mathrm{fs}$, which corresponds to the temporal resolution in the present experiment. About 10-times faster electron transfer compared to the relaxation time of hot electrons in $\mathrm{VSe}_{2}$ indicates that the photoexcited electrons in $\mathrm{VSe}_{2}$ are directly transferring into $\mathrm{Bi}_{2} \mathrm{Se}_{3}$ before thermalization owing to the fast decay channel of $\mathrm{Bi}_{2} \mathrm{Se}_{3}$ (Fig. 3c). This is consistent with the previously reported $\mathrm{VSe}_{2} /$ graphene heterostructures. ${ }^{[59]}$ Figure $3 \mathrm{c}$ shows the band diagram after junction in $\mathrm{VBH}$. The work function (W) of $\mathrm{VSe}_{2}$ is $5.8 \mathrm{eV}^{[62]}$ and the electron affinity $(\chi)$ of $\mathrm{Bi}_{2} \mathrm{Se}_{3}$ is $5.3 \mathrm{eV}^{[11]}$ with the bulk bandgap $\left(\mathrm{E}_{\mathrm{g}}\right.$, bulk $)$ of $0.3 \mathrm{eV}^{[14]}$, which creates an upward band bending of $\mathrm{Bi}_{2} \mathrm{Se}_{3}{ }^{[63]}$ and $n$-type junction with Schottky barrier $\left(\Phi_{\mathrm{SB}}\right)$ of about 0.5 $\mathrm{eV}$. Accordingly, the photoexcited electrons in $\mathrm{VSe}_{2}$ with a 1.5-eV optical pump can transfer energetically into $\mathrm{Bi}_{2} \mathrm{Se}_{3}$, which has a fast decay channel through the Dirac surface state, across the vdW gap. The observed larger magnitude $\Delta \mathrm{R} / \mathrm{R}_{0}$ and similar time constant of $\tau_{1}$ in VBH imply that the excited electrons in $\mathrm{VSe}_{2}$ can transfer to the bulk band in $\mathrm{Bi}_{2} \mathrm{Se}_{3}$ within an ultrashort time scale ( 100 fs), then efficiently relaxed by scattering with the Dirac surface state of $\mathrm{Bi}_{2} \mathrm{Se}_{3}$ as illustrated in Fig. 3c.

\section{Ultrafast carrier dynamics of insulating bulk surface states at VBHs}

After the intraband relaxation process, a relatively long lifetime of carriers in bulk band induced the derivative-like TR response, representing the optical transition in the bulk insulating band of $\mathrm{Bi}_{2} \mathrm{Se}_{3}$ that were maintained for several hundred ps. The increased reflectance (red color) at lower 
energy and decreased reflectance (blue color) at higher energy were observed at the boundary of $\sim 0.96 \mathrm{eV}$ in $\mathrm{Bi}_{2} \mathrm{Se}_{3}$ and VBH (Fig. 3a and Fig. 4a). The shape of TR spectra can be derived from the change of complex refractive index, which is closely related to the change of absorption coefficient as explained by the Kramers-Kronig relation. Accordingly, we fitted our experimentally observed TR spectra by taking into account the pump-induced refractive index changes of bulk band $\mathrm{Bi}_{2} \mathrm{Se}_{3}$ (details in Experimental Methods). This model provides information on the optical transition in $\mathrm{Bi}_{2} \mathrm{Se}_{3}$ such as the transition energy $\left(E_{\text {opt }}\right)$ and phenomenological broadening factor $(\Gamma)$. The fit results of TR spectra at 25 ps are displayed in Fig. $4 \mathrm{~b}$ and their photoinduced absorption changes $(\Delta \alpha)$ in Fig. $4 \mathrm{c}$ with the optical transition energy of $E_{o p t}=0.96$ $\mathrm{eV}$ and the broadening factor of $\Gamma=45 \mathrm{meV}$. The calculated absorption coefficient was subsequently confirmed by the transient absorption (TA) spectra and well-matched with the measured result as shown in Figs. S3b, c. According to the fitting model, an assumption of photoexcited carrier concentration $\left(n_{\mathrm{ex}}\right)$ of $9.7 \times 10^{18} \mathrm{~cm}^{-3}$, which is only $\sim 3 \%$ of the background carrier concentration $\left(N_{0}\right)$, provided the best fit of the spectral response at $25 \mathrm{ps}$. We note that the photoexcited carrier concentration of $9.7 \times 10^{18} \mathrm{~cm}^{-3}$ well matched the estimated value of $1.6 \times 10^{19}$ $\mathrm{cm}^{-3}$ from the complex refractive index of $\mathrm{Bi}_{2} \mathrm{Se}_{3}$ and optical power density, but was slightly small due to carrier recombination. The obtained transition energy of $0.96 \mathrm{eV}$ implied that the TR response originated from the pump-induced absorption between the split conduction bands $\left(\mathrm{CB}_{1-}\right.$ $\mathrm{CB}_{2}$ ) due to spin-orbit coupling. ${ }^{[64]}$ Additionally, the obtained transition energy of $0.96 \mathrm{eV}$ agreed well with the previous results of the bulk band in $\mathrm{Bi}_{2} \mathrm{Se}_{3} .{ }^{[64,65]}$

In the VBHs with 48, 57, 118, and $176 \mathrm{~nm}$-thick $\mathrm{VSe}_{2}$, the changes of the spectral and dynamical response of $\mathrm{Bi}_{2} \mathrm{Se}_{3}$ were observed according to $\mathrm{VSe}_{2}$ thickness as shown in Figs. 4a,b. A notable change is that the derivative-like $\mathrm{TR}$ of $\mathrm{Bi}_{2} \mathrm{Se}_{3}$ feature was redshifted with increasing 
thickness of $\mathrm{VSe}_{2}$, as indicated by the horizontal line in Fig. 4a and the asymmetry TR spectra (larger negative $\Delta R / R_{0}$ than positive $\Delta R / R_{0}$ ) became severe with increasing $V_{S e}$ thickness (Fig. 4b). This asymmetric behavior of the TR spectra in the VBHs originated from the TR component of $\mathrm{VSe}_{2}$, which remained almost constant (Fig. 3a and Figs. S4c). We took the TR response of $\mathrm{VSe}_{2}$ into account as a constant value during the model fitting process. Further details of TR spectrum modeling in VBHs can be found in the Experimental Methods section. The fitting result with our theoretical model provides the $E_{\text {opt }}$ and $\Gamma$ of photoinduced absorption changes in the VBHs as shown in Fig. 4c. The magnitude of $\Delta \alpha$ is significantly increased in VBHs ( 2.5 times in $167 \mathrm{~nm} \mathrm{VBH}$ ), which is consistent with the results shown in Fig. $3 \mathrm{~b}$ as the larger excited carrier density $\left(n_{e x}\right)$ by transferring from $\mathrm{VSe}_{2}$ to $\mathrm{Bi}_{2} \mathrm{Se}_{3}$. $E_{\text {opt }}$ shows a linear dependence on the thickness of $\mathrm{VSe}_{2}$ with the slope of $0.26 \mathrm{eV} / \mathrm{nm}$ (Fig. 4d). The factor of $\Gamma$ also tends to increase with the thickness of $\mathrm{VSe}_{2}$ (Fig. 4e). Figure 4f shows the decay characteristics of photoexcited carriers in the VBHs. In $\mathrm{Bi}_{2} \mathrm{Se}_{3}$, the pump-probe trace followed a biexponential decay. The fast decay time $\left(\tau_{1}\right)$ corresponds to the phonon-mediated bulk-surface scattering of excited carriers in $\mathrm{Bi}_{2} \mathrm{Se}_{3}$. The slow decay time ( $\left.\tau_{2}\right)$ of $\sim 600 \mathrm{ps}$ is attributed to continuously feeding the Dirac surface state and carrier recombination of metastable excited carrier population in the bulk band. ${ }^{[40,66]}$ The overall decay times are displayed in Fig. S5. The $\tau_{1}$ is almost constant with $\mathrm{VSe}_{2}$, which implies the maintenance of the Dirac surface state despite the presence of $\mathrm{VSe}_{2}$ junctions. But, the $\tau_{2}$ of $\sim 200$ ps in the $167 \mathrm{~nm}$ VBH is significantly fast, as shown in the inset of Fig. 4a. $\tau_{2}$ also showed a $\mathrm{VSe}_{2}$ thickness dependence, but inversely.

Such spectral and dynamical changes of derivative TR spectra of the insulating bulk band suggest interactions between metallic $\mathrm{VSe}_{2}$ and conduction/valence bands in $\mathrm{Bi}_{2} \mathrm{Se}_{3}$ by forming junctions. Accordingly, this section mainly handles the electronic interaction between the 
insulating bulk band of $\mathrm{Bi}_{2} \mathrm{Se}_{3}$ and metallic $\mathrm{VSe}_{2}$, which provides a good example of van der Waals MSJs. Previous ultrafast spectroscopic studies show that the interesting features in MSJ are determined by the interface. ${ }^{[34,35,67,68]}$ Generally, certain changes in the electronic states of the semiconductor in MSJs can be explained by metal/defect-induced gap states (MIGS, DIGS), or interfacial dipoles that cause the FLP observed in different MSJs. ${ }^{[1,22,68,69]}$ Since the MIGS store the electrons and holes and then pin the Fermi level, they can modulate the carrier dynamics of $\mathrm{Bi}_{2} \mathrm{Se}_{3}$. However, this can be sufficiently suppressed in the case of vdW MSJs owing to the vdW interaction. ${ }^{[18,20-22,29,30]}$ Although DIGS can also affect the carrier responses, similar to MIGS, they are negligible in the present work due to the verified high-quality interface. Most noteworthy is that both MIGS and DIGS, due to the overlap of wave functions occurring at the MS interface, are independent of the thickness of the contact metal. Consequently, the observed VSe 2 -thicknessdependent redshift of transition energy suggests that the bulk band modulation can be much better interpreted by interfacial dipole interaction rather than MIGS and DIGS in MSJ.

In the case of interfacial dipoles due to charge transfer and redistribution, they can shift the electronic level, deviating from the Schottky-Mott limit. ${ }^{[70-73]}$ Figure 5a illustrates the band alignment of isolated $\mathrm{VSe}_{2}$ and $\mathrm{Bi}_{2} \mathrm{Se}_{3}$ before contact. Due to the low $\chi(\sim 5.3 \mathrm{eV})$ and small bulk bandgap $\left(\mathrm{E}_{\mathrm{g}} \sim 0.3 \mathrm{eV}\right)$ in $\mathrm{Bi}_{2} \mathrm{Se}_{3}$ compared to the $W$ of $\mathrm{VSe}_{2}(\sim 5.8 \mathrm{eV})$, electrons are transferred from $\mathrm{Bi}_{2} \mathrm{Se}_{3}$ to $\mathrm{VSe}_{2}$ due to the Fermi level alignment after contact (Fig. 5a). Subsequently, the $\mathrm{VSe}_{2}$ layer close to the interface becomes negatively charged, while positive charges are created in the $\mathrm{Bi}_{2} \mathrm{Se}_{3}$ layer close to the interface, leading to interfacial dipoles (Fig. 5a). These interfacial dipoles due to charge transfer lift the electronic level of $\mathrm{Bi}_{2} \mathrm{Se}_{3}$ layers near the interface, whereas the $\mathrm{Bi}_{2} \mathrm{Se}_{3}$ layers far from the interface remain unchanged due to the rapid decay with distance from the interface. ${ }^{[70,72]}$ With this modulation, a band offset $(\Delta)$ is created as displayed in Fig. 5a. 
We note that the photoinduced built-in electric field is negligible at the interface of the MSJ in our experiment due to a small perturbation of $\sim 3 \%$ of background carrier density. Hence, dipole interactions is dominant, which enables us to estimate the $\Delta$ created by the junction with metallic $\mathrm{VSe}_{2}$. The redshift of the optical transition energy in the VBHs makes it possible to move the transitions of $\mathrm{CB}_{1}-\mathrm{CB}_{2}$ to promoted by $\Delta$. Thus, the observed transition energy directly reflects the $\Delta$ induced by the interfacial dipole interaction with the electronic level of $\mathrm{Bi}_{2} \mathrm{Se}_{3}$. Moreover, the gradual change in the electronic level of $\mathrm{Bi}_{2} \mathrm{Se}_{3}$ layers also resulted in the broadening of the projected $\mathrm{Bi}_{2} \mathrm{Se}_{3}$ band structure. The effect through the interfacial dipole interaction depends on the thickness of the metallic contact layer because the transfer of a large number of electrons requires Fermi level alignment with increasing $\mathrm{VSe}_{2}$ thickness, and thus, the dipole strength becomes stronger (Fig. 5a). ${ }^{[73,74]}$ Particularly, in the 167 nm-thick VBH (dashed box in Fig. 4a), the observed additional optical transition over $1 \mathrm{eV}$ reflects the highly distorted electronic level due to strong dipole interactions. However, the redshift tendency corresponding to the dependence on the $\mathrm{VSe}_{2}$ thickness remains for the transition energy of interest. Note that the electronic level shift of $\mathrm{Bi}_{2} \mathrm{Se}_{3}$ by the junction with $\mathrm{VSe}_{2}$ was $\sim 10$ times lower compared to conventional 3D metals, ${ }^{[11]}$ including Au, Pd, and Pt. This implies that extremely weak interactions can be achieved with $\mathrm{VSe}_{2}$ as the $2 \mathrm{D}$ metal electrode.

The estimated $\Delta$ showed a linear dependence on the thickness of $\mathrm{VSe}_{2}$ with a slope of 0.26 $\mathrm{meV} / \mathrm{nm}$ (Fig. 5b). The $\Delta$ on the order of several tenths of meV is comparable to the order of the first-principles calculation result for metal-2D semiconductor junctions. ${ }^{[72]}$ Since $\Phi_{\mathrm{SB}}$ is determined by the energy difference between the conduction band edges and the Fermi level in $\mathrm{Bi}_{2} \mathrm{Se}_{3}\left(\Phi_{\mathrm{SB}}=\mathrm{E}_{\mathrm{CBM}}-\mathrm{E}_{\mathrm{F}}\right)$ for electrons, the $\Delta$ observed from their inherent levels leads to a deviation from the Schottky-Mott limit $(\mathrm{S}=1) \cdot{ }^{[22,70-73]}$ The pinning factor $\mathrm{S}$, which is defined as 
$S=\left|d \Phi_{B} / d W\right|$ and denotes the strength of FLP, which is deduced from the estimated $\Delta$ (Fig. 5c). Note that $\mathrm{S}$ became closer to the Schottky-Mott limit as the thickness of $\mathrm{VSe}_{2}$ decreased. Consequently, the changes in the SBH due to charge transfer and interfacial dipoles are expected to be weak in vdW MSJs with an ultrathin layer (monolayer to few-layer), and further approaching the ideal Schottky-Mott limit $(\mathrm{S}=1)$ is possible. The broadening factor of the optical transition in $\mathrm{Bi}_{2} \mathrm{Se}_{3}$ also increased with the increasing thickness of the $\mathrm{VSe}_{2}$ layer (Fig. 4e). The gradual shift of the electronic bands in the $\mathrm{Bi}_{2} \mathrm{Se}_{3}$ layers reflects that the width of the optical transition in $\mathrm{Bi}_{2} \mathrm{Se}_{3}$ is broadened in the projected band structure, as illustrated in Fig. 5a. This trend was also observed in previous studies on electronic band modulation under an external electric field. ${ }^{[75-77]}$ Moreover, since the lifetime of metastable carriers $\left(\tau_{2}\right)$ strongly depends on the Fermi level of $\mathrm{Bi}_{2} \mathrm{Se}_{3},{ }^{[41-42]}$ strong FLP can pin the $\tau_{2}$ regardless of $\mathrm{VSe}_{2}$ thickness. Accordingly, significant $\mathrm{VSe}_{2}$ thicknessdependence of $\tau_{2}$ shows the additional evidence of weak FLP, originated by interfacial hybridization or defect states. On the other side, the interband recombination time of metastable carrier can be decreased as $\Delta$ increased owing to the interfacial dipole interaction. Under $\Delta$, the electrons and holes were driven in opposite directions (Fig. 5a). Then, it becomes possible for the metastable carriers of $\mathrm{Bi}_{2} \mathrm{Se}_{3}$ to flow efficiently to each surface, giving rise to the shortening of the metastable carrier lifetime (Fig. 4f). This result is consistent with the observed redshift of the transition energy and broadening of the optical transition and shows good agreement with previous reports on exciton dissociation mechanisms, ${ }^{[70]}$ which were previously studied in 2D semiconductors with in-plane dissociation of strongly bound exciton under an external electric field ${ }^{[75]}$ and out-of-plane dissociation due to the built-in-field of the $p-n$ doping ${ }^{[78]}$. 


\section{CONCLUSION}

In summary, by employing ultrafast spectroscopy, we investigated the interfacial carrier dynamics in both of metallic surface state and insulating bulk state in $\mathrm{VSe}_{2} / \mathrm{Bi}_{2} \mathrm{Se}_{3}$, for which a nearly perfect interface was realized and the dependence of the dipole effect hidden by MIGS and DIGS on the metal thickness was observed. The excellent interfacial contact in the MSJs was verified by precise STEM images and interfacial CAP dynamics. The hot electron transfer from $\mathrm{VSe}_{2}$ to $\mathrm{Bi}_{2} \mathrm{Se}_{3}$ across $\mathrm{vdW}$ gap in ultrashort timescale ( $\sim 100 \mathrm{fs}$ ) before thermalization in $\mathrm{VSe}_{2}$ enables to improve the efficiency of $\mathrm{Bi}_{2} \mathrm{Se}_{3}$-based optoelectronic devices. Furthermore, it was found that the metallic surface state of $\mathrm{Bi}_{2} \mathrm{Se}_{3}$ was well preserved even after contact with $\mathrm{VSe}_{2}$. The $\mathrm{VSe}_{2}$ thicknessdependent TR measurements quantitatively provided electronic level shifts of the bulk band in $\mathrm{Bi}_{2} \mathrm{Se}_{3}$ due to dipole interactions, leading to deviation from the Schottky-Mott limit. In addition, the observed carrier long-decay characteristics in VBHs show a strong dependence on the $\mathrm{VSe}_{2}$ thickness and there were no significant FLP effects. The metastable carrier lifetime was noticeably shorter due to the built-in field, enabling a highly efficient current flow of $\mathrm{Bi}_{2} \mathrm{Se}_{3}$-based devices.

Our results show that $\mathrm{VSe}_{2}$ is a promising candidate as the $2 \mathrm{D}$ metal electrode for diverse applications based on $\mathrm{Bi}_{2} \mathrm{Se}_{3}$ and other topological insulators. Moreover, the interfacial dipole interaction created by charge transfer and redistribution allows us to understand the carrier dynamics and modulated electronic states in MSJs, approaching the ideal Schottky-Mott limit. Although thickness control of the semiconductor part in MSJs has been suggested to cover the effects of interfacial dipoles due to the depinning effect between the semiconductor layers, i.e., a multilayer (thick) semiconductor is an advantageous option for suppressing FLP, ${ }^{[70,72]}$ the practical development remains elusive due to the significant changes in the electrical and optical properties from the monolayer counterpart. Thus, the combination of defining a new approach to perceive 
the modulated band structures in MSJs that may encompass delicate materials and finding suitable $\mathrm{vdW}$ materials close to the ideal junctions shown in the present work provides a new strategy for the contact fabrication process and will open up unprecedented opportunities for optimizing device performance by fully utilizing the intrinsic properties of $\mathrm{Bi}_{2} \mathrm{Se}_{3}$ and other topological insulators. 


\section{METHODS}

\section{Fabrication of $\mathrm{VSe}_{2} / \mathrm{Bi}_{2} \mathrm{Se}_{3}$ heterostructures}

$\mathrm{A} \mathrm{Bi}_{2} \mathrm{Se}_{3}$ thin film was grown by a molecular beam epitaxy system with an ultrahigh vacuum chamber equipped with VEECO (Knudsen) cells of high purity bismuth (99.999+\%) and selenium (99.999\%) sources. ${ }^{[79]}$ Prior to growth, the $\mathrm{Al}_{2} \mathrm{O}_{3}$ (001) substrate was cleaned with acetone and isopropyl alcohol and baked at $700^{\circ} \mathrm{C}$ for 30 min to remove any residual contaminants. A $\mathrm{Bi}_{2} \mathrm{Se}_{3} / \mathrm{Al}_{2} \mathrm{O}_{3}$ (001) film was grown at $250^{\circ} \mathrm{C}$ for $90 \mathrm{~min}$, followed by a post-annealing process of $450^{\circ} \mathrm{C}$ for $30 \mathrm{~min}$ for self-crystallization. The ratio of $\mathrm{Bi}$ and Se fluxes was kept at $\sim 1: 15$ to minimize Se vacancies. The formation of crystalline $\mathrm{Bi}_{2} \mathrm{Se}_{3}$ on the substrate was monitored by an in situ reflections high-energy electron diffraction system. The carrier concentration was determined by Hall resistivity measurement at $300 \mathrm{~K}$. The carrier concentration $\left(N_{0}\right)$ of $\mathrm{Bi}_{2} \mathrm{Se}_{3}$ is given by $N_{0}=(B \cdot I) /\left(e \cdot d \cdot V_{\mathrm{H}}\right)$, where $I$ is current, $d$ is the thickness of the $\mathrm{Bi}_{2} \mathrm{Se}_{3}$ thin film, $e$ is the electron charge, and $V_{\mathrm{H}}$ is the Hall voltage; thus, we can estimate $N_{0}=3.13 \times 10^{20} \mathrm{~cm}^{-3}$.

Single crystalline $1 \mathrm{~T}-\mathrm{VSe}_{2}$ was grown by the chemical vapor transport method. ${ }^{[43]} \mathrm{V}$ (99.5\% purity) and $\mathrm{Se}$ (99.999\% purity) powders were used as the precursors with a stoichiometric amount of 2:1 wt $\%$. As a transport agent (concentration of $1.5 \mathrm{mg} / \mathrm{cm}^{3}$ ), $\mathrm{VCl}_{3}$ powder (99\% purity) was used to provide sufficient vapor pressure. The mixture of precursors and transport agents was placed in a quartz ampule. The ampule was evacuated below $10^{-6}$ Torr, followed by melting and sealing. The sealed quartz ampule was placed at the centre between two heating zones, which were heated slowly in five steps to approach their target temperatures $\left(750^{\circ} \mathrm{C}\right.$ for vaporization and $850^{\circ} \mathrm{C}$ for growth). After being held for $72 \mathrm{~h}$ at the maximum temperatures, the system was cooled down naturally, and shiny silver-grey $\mathrm{VSe}_{2}$ crystals were collected after breaking the ampule. 
We adopted mechanical exfoliation to transfer $\mathrm{VSe}_{2}$ onto $\mathrm{Bi}_{2} \mathrm{Se}_{3}$ for the fabrication of $\mathrm{VSe}_{2} / \mathrm{Bi}_{2} \mathrm{Se}_{3}$ heterostructures. VSe $\mathrm{V}_{2}$ flakes were transferred onto the $\mathrm{Bi}_{2} \mathrm{Se}_{3}$ thin film by using a simple blue tape method. We selected one of them, and different thicknesses were exposed on one $\mathrm{VSe}_{2}$ flake for a consistent experimental environment.

\section{Characterization of $\mathrm{VSe}_{2} / \mathrm{Bi}_{2} \mathrm{Se}_{3}$ heterostructures}

The thickness of the $\mathrm{VSe}_{2}$ flake was analyzed by AFM (n-Tracer, Nano Focus Inc.) operated in a non-contact mode. Raman measurements were performed with a Raman spectrometer (FEX, NOST) by focusing the laser radiation with a $0.3 \mathrm{~mW}$ power centred at $531 \mathrm{~nm}$. A microstructural study was carried out using a STEM system (JEM-ARM200F, JEOL) equipped with a probe spherical aberration corrector. HAADF-STEM images were obtained to confirm the atomic structure in real space. For HAADF-STEM images, the convergent semiangle was fixed at $22 \mathrm{mrad}$, and the collection semiangle was $67-170 \mathrm{mrad}$. TEM specimens were thinned for electron transparency by a focused ion beam system.

\section{Transient reflectance (TR) measurements}

CAP dynamics and TR measurements were performed by employing a Ti:sapphire laser oscillator (MAITAI, Spectra-Physics), which produced 100-fs pump pulses at an 80-MHz repetition rate. The main portion of the output from the oscillator at a central wavelength of $830 \mathrm{~nm}$ was further used to pump a synchronously pumped optical parametric oscillator (SPOPO) for the generation of tuneable 150 -fs probe pulses $(1150-1550 \mathrm{~nm})$ at the same repetition rate. With a dichroic mirror, the pump and probe pulses were collinearly combined and focused on the sample to a beam waist of $\sim 2 \mu \mathrm{m}$ by a single objective lens (M Plan Apo 100×, Mitutoyo). The pump fluence 
adjusted with two polarizers was $\sim 12 \mu \mathrm{J} / \mathrm{cm}^{2}$. The time delay between the pump and probe pulses was controlled by using a motorized linear stage. The reflected probe beam was recorded with a Ge photodetector (DET50B, Thorlabs) connected to a lock-in amplifier (SR830, Stanford Research Systems) after blocking the reflected pump beams. Based on this setup, we measured the time-

resolved differential reflectance defined by $\frac{\Delta R}{R_{0}} \equiv \frac{R_{0}-R^{\prime}(t)}{R_{0}}$, where $R_{0}$ and $R^{\prime}(t)$ are the reflectance of the sample without and with pump excitation, respectively. All measurements were carried out under ambient conditions at room temperature.

\section{Modelling of TR spectra}

The theoretical model for analyzing the TR feature is based on pump-induced refractive index changes for semiconducting materials. ${ }^{[36,57]}$ The pump pulses excite electrons in that ground state, then perturb the dielectric response and fractionally change the complex refractive index of $\tilde{n}=$ $n+i k$. First, we calculated the imaginary part of $k$ corresponding to absorption coefficient as $k=(\lambda / 4 \pi) \alpha$, where $\lambda$ is the probe wavelength and $\alpha$ is the absorption coefficient. Once the change of absorption coefficient $(\Delta \alpha)$ is obtained, the change of real part of the refractive index $(\Delta n)$ is derived by using the Kramers-Kronig transformation

$$
\Delta \mathrm{n}(\hbar \omega)=\frac{\hbar c}{\pi} P \int_{0}^{\infty} \frac{\Delta \alpha(\hbar \omega)}{\omega^{\prime 2}-\omega^{2}} d \omega^{\prime},
$$

where $c$ is the speed of light, and $P$ is the Cauchy principal value for the integral. We calculated the absorption coefficient change with optical absorption of $\delta\left(\hbar \omega_{\text {opt }}\right)$ with perturbed factor as photoexcited carrier concentration divided by doping concentration $\left(\Delta N / N_{0}\right)$ and with homogeneous broadening by deconvolution with Gaussian function. The broadening factor $\Gamma$ is included in the calculation. 
In the measurement, since the observed fractional reflection change $\left(\Delta R / R_{0}\right)$ was too small $\left(\sim 10^{-4}\right)$ by normal incident pump pulses, the observed differential reflection was dominated by refractive index changes. From this, $\Delta R / R_{0}$ can be approximated as

$$
\frac{\Delta R}{R_{0}}(\hbar \omega) \approx\left(\frac{4}{n(\hbar \omega)^{2}-1}\right) \cdot \Delta n(\hbar \omega),
$$

where $n_{0}=5.5$ is the averaged refractive index of $\mathrm{Bi}_{2} \mathrm{Se}_{3}$ in the interesting spectral region. ${ }^{\left[{ }^{[80]}\right.} \mathrm{To}$ obtain the optical transition energy and broadening factor in VBHs, we performed the model fit in TR spectra of VBH at the same time delay of $25 \mathrm{ps}$. In $\mathrm{Bi}_{2} \mathrm{Se}_{3}$, asymmetrical positive and negative peaks in TR spectra were observed as displayed in Fig. S3c, S4c and S4d. However, in VBHs, the asymmetrical TR spectra were observed with small positive peaks at lower energy and large negative peaks at higher energy as can be seen in Fig. 3e. Subsequently, we modified the equation (3) for TR spectra in VBHs as

$$
\frac{\Delta R}{R_{0}}(\hbar \omega) \approx\left(\frac{4}{n(\hbar \omega)^{2}-1}\right) \cdot \Delta n(\hbar \omega)+C_{V S e_{2}}
$$

where $C_{\mathrm{VSe}_{2}}$ is the constant TR response of the bare $\mathrm{VSe}_{2}$ as shown in Fig. S4c. As discussed in the main text, the optical transition energy was redshifted and the broadening factor became larger by the contact with $\mathrm{VSe}_{2}$. The value of $C_{V S e_{2}}$ became larger with the increase of $\mathrm{VSe}_{2}$ thickness and is consistent with the modified model of TR response in VBHs. 


\section{DATA AVAILABILITY}

Data are available on request from the authors.

\section{REFERENCES}

1. Moore, J. E. The birth of topological insulators. Nature 464, 194-198 (2010).

2. Hasan, M. Z. \& Kane, C. L. Colloquium: topological insulators. Rev. Mod. Phys. 82, 3045 (2010).

3. Pesin, D. \& MacDonald, A. H. Spintronics and pseudospintronics in graphene and topological insulators. Nat. Mater. 11, 409-416 (2012).

4. Vandenberghe, W. G. \& Fischetti, M. V. Imperfect two-dimensional topological insulator field-effect transistors. Nat. Commun. 8, 1-8 (2017).

5. Zhang, Q., Iannaccone, G. \& Fiori, G. Two-Dimensional Tunnel Transistors Based on $\mathrm{Bi}_{2} \mathrm{Se}_{3}$ Thin Film. IEEE Electron Device Lett. 35, 129-131 (2013).

6. McIver, J., Hsieh, D., Steinberg, H., Jarillo-Herrero, P. \& Gedik, N. Control over topological insulator photocurrents with light polarization. Nat. Nanotechnol. 7, 96-100 (2012).

7. Huang, S. \& Xu, X. Optical Chirality Detection Using a Topological Insulator Transistor. $A d v$. Opt. Mater. 9, 2002210 (2021).

8. Zhang, H., Zhang, X., Liu, C., Lee, S.-T. \& Jie, J. High-responsivity, high-detectivity, ultrafast topological insulator $\mathrm{Bi}_{2} \mathrm{Se}_{3} /$ silicon heterostructure broadband photodetectors. ACS Nano 10, 5113-5122 (2016).

9. Wang, F. et al. Submillimeter $2 \mathrm{D} \quad \mathrm{Bi}_{2} \mathrm{Se}_{3}$ Flakes toward High-Performance Infrared Photodetection at Optical Communication Wavelength. Adv. Funct. Mater. 28, 1802707 (2018).

10. Mellnik, A. et al. Spin-transfer torque generated by a topological insulator. Nature 511, 449451 (2014).

11. Spataru, C. D. \& Léonard, F. Fermi-level pinning, charge transfer, and relaxation of spinmomentum locking at metal contacts to topological insulators. Phys. Rev. B 90, 085115 (2014).

12. Walsh, L. A. et al. Interface chemistry of contact metals and ferromagnets on the topological insulator $\mathrm{Bi}_{2} \mathrm{Se}_{3}$. J. Phys. Chem. C 121, 23551-23563 (2017). 
13. Yeh, Y.-C. et al. Growth of the Bi2Se3 Surface Oxide for Metal-Semiconductor-Metal Device Applications. J. Phys. Chem. C 120, 3314-3318 (2016).

14. Cho, S., Butch, N. P., Paglione, J. \& Fuhrer, M. S. Insulating behavior in ultrathin bismuth selenide field effect transistors. Nano Lett. 11, 1925-1927 (2011).

15. Lang, M. et al. Revelation of topological surface states in $\mathrm{Bi}_{2} \mathrm{Se}_{3}$ thin films by in situ $\mathrm{Al}$ passivation. ACS Nano 6, 295-302 (2012).

16. Xu, S. et al. van der Waals epitaxial growth of atomically thin $\mathrm{Bi}_{2} \mathrm{Se}_{3}$ and thickness-dependent topological phase transition. Nano Lett. 15, 2645-2651 (2015).

17. Kim, C. et al. Fermi level pinning at electrical metal contacts of monolayer molybdenum dichalcogenides. ACS Nano 11, 1588-1596 (2017).

18. Wang, Y. et al. Van der Waals contacts between three-dimensional metals and twodimensional semiconductors. Nature 568, 70-74 (2019).

19. Jena, D., Banerjee, K. \& Xing, G. H. 2D crystal semiconductors: Intimate contacts. Nat. Mater. 13, 1076-1078 (2014).

20. Allain, A., Kang, J., Banerjee, K. \& Kis, A. Electrical contacts to two-dimensional semiconductors. Nat. Mater. 14, 1195-1205 (2015).

21. Liu, Y. et al. Approaching the Schottky-Mott limit in van der Waals metal-semiconductor junctions. Nature 557, 696-700 (2018).

22. Liu, Y., Stradins, P. \& Wei, S.-H. Van der Waals metal-semiconductor junction: Weak Fermi level pinning enables effective tuning of Schottky barrier. Sci. Adv. 2, e1600069 (2016).

23. Shen, T., Ren, J.-C., Liu, X., Li, S. \& Liu, W. van der Waals stacking induced transition from Schottky to ohmic contacts: 2D metals on multilayer InSe. J. Am. Chem. Soc. 141, 3110-3115 (2019).

24. Liu, Y. et al. Toward barrier free contact to molybdenum disulfide using graphene electrodes. Nano Lett. 15, 3030-3034 (2015).

25. Chuang, H.-J. et al. High mobility $\mathrm{WSe}_{2} p$-and $n$-type field-effect transistors contacted by highly doped graphene for low-resistance contacts. Nano Lett. 14, 3594-3601 (2014).

26. Chee, S. S. et al. Lowering the Schottky barrier height by graphene/Ag electrodes for highmobility $\mathrm{MoS}_{2}$ field-effect transistors. Adv. Mater. 31, 1804422 (2019).

27. Zhao, B. et al. 2D Metallic Transition-Metal Dichalcogenides: Structures, Synthesis, Properties, and Applications. Adv. Funct. Mater. 2105132 (2021).

28. Zhang, Z. et al. Van der Waals epitaxial growth of 2D metallic vanadium diselenide single crystals and their extra-high electrical conductivity. Adv. Mater. 29, 1702359 (2017).

29. Shi, J. et al. Two-Dimensional Metallic Vanadium Ditelluride as a High-Performance Electrode Material. ACS Nano 15, 1858-1868 (2021). 
30. Ji, Q. et al. Metallic vanadium disulfide nanosheets as a platform material for multifunctional electrode applications. Nano Lett. 17, 4908-4916 (2017).

31. Zhang, Z. et al. Epitaxial growth of two-dimensional metal-semiconductor transition-metal dichalcogenide vertical stacks $\left(\mathrm{VSe}_{2} / \mathrm{MX}_{2}\right)$ and their band alignments. ACS Nano 13, 885-893 (2018).

32. Ci, H. et al. Defective $\mathrm{VSe}_{2}-$ Graphene Heterostructures Enabling In Situ Electrocatalyst Evolution for Lithium-Sulfur Batteries. ACS Nano 14, 11929-11938 (2020).

33. Yang, C. et al. Metallic graphene-like $\mathrm{VSe}_{2}$ ultrathin nanosheets: superior potassium-ion storage and their working mechanism. Adv. Mater. 30, 1800036 (2018).

34. Yang, Y. et al. Semiconductor interfacial carrier dynamics via photoinduced electric fields. Science 350, 1061-1065 (2015).

35. Chen, X., Wang, K. \& Beard, M. C. Ultrafast probes at the interfaces of solar energy conversion materials. Phys. Chem. Chem. Phys. 21, 16399-16407 (2019).

36. Yang, Y. et al. Low surface recombination velocity in solution-grown $\mathrm{CH}_{3} \mathrm{NH}_{3} \mathrm{PbBr}_{3}$ perovskite single crystal. Nat. Commun. 6, 1-6 (2015).

37. Zhong, C. et al. Hot carrier and surface recombination dynamics in layered InSe crystals. $J$. Phys. Chem. Lett. 10, 493-499 (2019).

38. $\mathrm{Wu}, \mathrm{L}$. et al. A sudden collapse in the transport lifetime across the topological phase transition in $\left(\mathrm{Bi}_{1-\mathrm{x}} \mathrm{In}_{\mathrm{x}}\right)_{2} \mathrm{Se}_{3}$. Nat. Phys. 9, 410-414 (2013).

39. Glinka, Y. D. et al. Ultrafast carrier dynamics in thin-films of the topological insulator $\mathrm{Bi}_{2} \mathrm{Se}_{3}$. Appl. Phys. Lett. 103, 151903 (2013).

40. Glinka, Y. D., Li, J., He, T. \& Sun, X. W. Clarifying ultrafast carrier dynamics in ultrathin films of the topological insulator $\mathrm{Bi}_{2} \mathrm{Se}_{3}$ using transient absorption spectroscopy. ACS Photon. 8, 1191-1205 (2021).

41. Luo, C.-W. et al. Snapshots of Dirac fermions near the Dirac point in topological insulators. Nano Lett. 13, 5797-5802 (2013).

42. Sim, S. et al. Picosecond Competing Dynamics of Apparent Semiconducting-Metallic Phase Transition in the Topological Insulator $\mathrm{Bi}_{2} \mathrm{Se}_{3}$. ACS Photon. 7, 759-764 (2020).

43. Feroze, A. et al. In-Depth Structural Characterization of $1 \mathrm{~T}-\mathrm{VSe}_{2}$ Single Crystals Grown by Chemical Vapor Transport. Cryst. Growth Des. (2020).

44. Zhao, Y. et al. Interlayer vibrational modes in few-quintuple-layer $\mathrm{Bi}_{2} \mathrm{Te}_{3}$ and $\mathrm{Bi}_{2} \mathrm{Se}_{3}$ twodimensional crystals: Raman spectroscopy and first-principles studies. Phys. Rev. B 90, 245428 (2014).

45. Li, D. et al. Structural Phase Transition of Multilayer VSe2. ACS Appl. Mater. Interfaces 12, 25143-25149 (2020). 
46. Park, T. G. et al. Coherent Control of Interlayer Vibrations in $\mathrm{Bi}_{2} \mathrm{Se}_{3}$ van der Waals Thin-Films. Nanoscale, 2021, DOI: 10.1039/d1nr05075c.

47. Bayliss, S. \& Liang, W. Reflectivity and band structure of 1T-VSe 2. J. Phys. C: Solid State Phys. 17, 2193 (1984).

48. McIver, J. et al. Theoretical and experimental study of second harmonic generation from the surface of the topological insulator $\mathrm{Bi}_{2} \mathrm{Se}_{3}$. Phys. Rev. B 86, 035327 (2012).

49. Greener, J. D. et al. Coherent acoustic phonons in van der Waals nanolayers and heterostructures. Phys. Rev. B 98, 075408 (2018).

50. Glinka, Y. D., Babakiray, S., Johnson, T. A., Holcomb, M. B. \& Lederman, D. Acoustic phonon dynamics in thin-films of the topological insulator $\mathrm{Bi}_{2} \mathrm{Se}_{3}$. J. Appl. Phys. Lett. 117, 165703 (2015).

51. Wang, H., Xu, Y., Shimono, M., Tanaka, Y. \& Yamazaki, M. Computation of interfacial thermal resistance by phonon diffuse mismatch model. Mater. Trans. 48, 2349-2352 (2007).

52. Gospodarev, I. et al. Elastic properties and phonon spectra of quasi-two-dimensional $\mathrm{VSe}_{2}$. Low Temp. Phys. 29, 151-154 (2003).

53. Greener, J. D. et al. High-Frequency Elastic Coupling at the Interface of van der Waals Nanolayers Imaged by Picosecond Ultrasonics. ACS Nano 13, 11530-11537 (2019).

54. Wen, Y.-C. et al. Specular scattering probability of acoustic phonons in atomically flat interfaces. Phys. Rev. Lett. 103, 264301 (2009).

55. Sobota, J. A. et al. Ultrafast optical excitation of a persistent surface-state population in the topological insulator $\mathrm{Bi}_{2} \mathrm{Se}_{3}$. Phys. Rev. Lett. 108, 117403 (2012).

56. Sim, S. et al. Ultrafast terahertz dynamics of hot Dirac-electron surface scattering in the topological insulator $\mathrm{Bi}_{2} \mathrm{Se}_{3}$. Phys. Rev. B 89, 165137 (2014).

57. Bennett, B. R., Soref, R. A. \& Del Alamo, J. A. Carrier-induced change in refractive index of InP, GaAs and InGaAsP. IEEE J. Quantum Electron. 26, 113-122 (1990).

58. Li, F., Tu, K. \& Chen, Z. Versatile electronic properties of $\mathrm{VSe}_{2}$ bulk, few-layers, monolayer, nanoribbons, and nanotubes: A computational exploration. J. Phys. Chem. C 118, 2126421274 (2014).

59. Park, T. G. et al. Interlayer coupling and ultrafast hot electron transfer dynamics in metallic $\mathrm{VSe}_{2}$ /graphene van der Waals heterostructures. ACS Nano 15, 7756-7764 (2021).

60. He, J. et al. Electron transfer and coupling in graphene-tungsten disulfide van der Waals heterostructures. Nat. Commun. 5, 1-5 (2014).

61. Yuan, L. et al. Photocarrier generation from interlayer charge-transfer transitions in $\mathrm{WS}_{2}$ graphene heterostructures. Sci. Adv. 4, e1700324 (2018). 
62. Claessen, R., Schafer, I. \& Skibowski, M. The unoccupied electronic structure of 1T-VSe $2 . J$. Phys.: Condens. Matter 2, 10045 (1990).

63. Brahlek, M., Kim, Y. S., Bansal, N., Edrey, E. \& Oh, S. Surface versus bulk state in topological insulator $\mathrm{Bi}_{2} \mathrm{Se}_{3}$ under environmental disorder. Appl. Phys. Lett. 99, 012109 (2011).

64. Zhang, H. et al. Topological insulators in $\mathrm{Bi}_{2} \mathrm{Se}_{3}, \mathrm{Bi}_{2} \mathrm{Te}_{3}$ and $\mathrm{Sb}_{2} \mathrm{Te}_{3}$ with a single Dirac cone on the surface. Nat. Phys. 5, 438-442 (2009).

65. Lawal, A. \& Shaari, A. Density functional theory study of electronic properties of $\mathrm{Bi}_{2} \mathrm{Se}_{3}$ and $\mathrm{Bi}_{2} \mathrm{Te}_{3}$. Mal. J. Fund. Appl. Sci. 12 (2016).

66. Glinka, Y. D., Babakiray, S., Johnson, T. A., Holcomb, M. B. \& Lederman, D. Effect of carrier recombination on ultrafast carrier dynamics in thin films of the topological insulator $\mathrm{Bi}_{2} \mathrm{Se}_{3}$. Appl. Phys. Lett. 105, 171905 (2014).

67. Lee, K. et al. Sub-picosecond carrier dynamics induced by efficient charge transfer in $\mathrm{MoTe}_{2} / \mathrm{WTe}_{2}$ van der Waals heterostructures. ACS Nano 13, 9587-9594 (2019).

68. Seo, D. M. et al. Ultrafast Excitonic Behavior in Two-Dimensional Metal-Semiconductor Heterostructure. ACS Photon. 6, 1379-1386 (2019).

69. Went, C. M. et al. A new metal transfer process for van der Waals contacts to vertical Schottkyjunction transition metal dichalcogenide photovoltaics. Sci. Adv. 5, eaax6061 (2019).

70. Wang, Q., Shao, Y. \& Shi, X. Mechanism of charge redistribution at the metal-semiconductor and semiconductor-semiconductor interfaces of metal-bilayer $\mathrm{MoS}_{2}$ junctions. J. Chem. Phys. $152,244701(2020)$.

71. Tung, R. T. The physics and chemistry of the Schottky barrier height. Appl. Phys. Rev. 1, 011304 (2014).

72. Wang, Q., Shao, Y., Gong, P. \& Shi, X. Metal-2D multilayered semiconductor junctions: layer-number dependent Fermi-level pinning. J. Mater. Chem. C 8, 3113-3119 (2020).

73. Tung, R. T. Formation of an electric dipole at metal-semiconductor interfaces. Phys. Rev. B 64, 205310 (2001).

74. Reusch, T., Wenderoth, M., Winking, L., Quaas, N. \& Ulbrich, R. Origin of Schottky Barriers in Gold Contacts on GaAs (110). Phys. Rev. Lett. 93, 206801 (2004).

75. Massicotte, M. et al. Dissociation of two-dimensional excitons in monolayer $\mathrm{WSe}_{2}$. Nat. Coтmun. 9, 1-7 (2018).

76. Zhang, L. et al. Quantum-confined stark effect in the ensemble of phase-pure CdSe/CdS quantum dots. Nanoscale 11, 12619-12625 (2019).

77. Park, K., Deutsch, Z., Li, J. J., Oron, D. \& Weiss, S. Single molecule quantum-confined Stark effect measurements of semiconductor nanoparticles at room temperature. ACS Nano 6, 1001310023, (2012). 
78. Riis-Jensen, A. C., Pandey, M. \& Thygesen, K. S. Efficient charge separation in 2D Janus van der Waals structures with built-in electric fields and intrinsic $p-n$ doping. J. Phys. Chem. C 122, 24520-24526 (2018).

79. Jerng, S.-K., Jeon, J. H., Kim, Y., Kim, J. S. \& Chun, S.-H. Multiple surface conduction channels via topological insulator and amorphous insulator thin film multi-stacks. Curr. Appl. Phys. 19, 219-223 (2019).

80. Eddrief, M., Vidal, F. \& Gallas, B. Optical properties of $\mathrm{Bi}_{2} \mathrm{Se}_{3}$ : from bulk to ultrathin films. J. Phys. D: Appl. Phys. 49, 505304 (2016). 


\section{ACKNOWLEDGMENT}

This work was supported by the National Research Foundation of Korea (NRF) funded by the Korean Government (2019R1A2C3003504, 2020R1A4A2002828). J.H.J. and S.-H.C. acknowledge the support from the NRF grant (2016R1E1A1A01942649). S.L acknowledges the support from the NRF grant (2021R1F1A1050726).

\section{AUTHOR CONTRIBUTIONS}

T.G.P., S.L., and F.R. conceived the original idea. J.H.J., S.-H.C., and S.L. synthesized and characterized the $\mathrm{VSe}_{2} / \mathrm{Bi}_{2} \mathrm{Se}_{3}$ heterostructure samples. T.G.P. performed the time-resolved experiments and data analysis. T.G.P. S.L. and F.R. discussed the results. F.R. supervised the project. T.G.P., S.L., and F.R. wrote the manuscript with input from all authors.

\section{COMPETING INTERESTS}

The authors declare no competing interests.

\section{ADDITIONAL INFORMATION}

Supplementary Information The Supplementary Information is available at http://..

Correspondence and requests for materials should be addressed to S. L, F. R. 


\section{Figures}

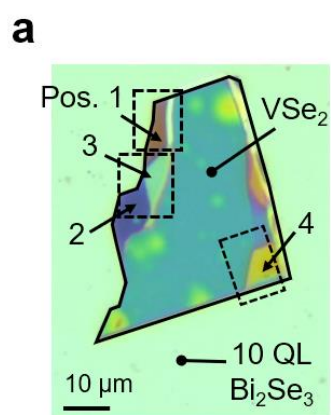

b

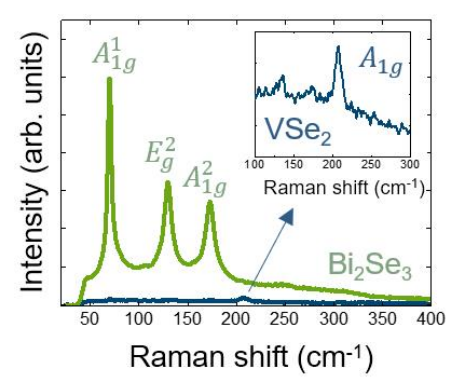

d
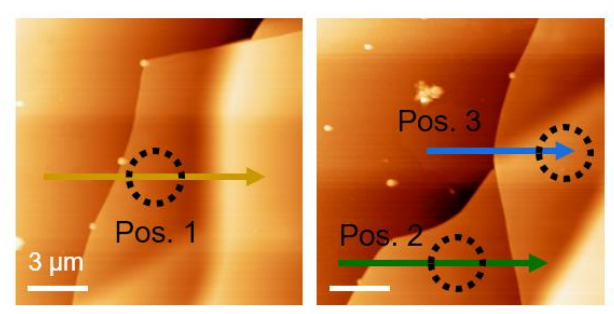

C

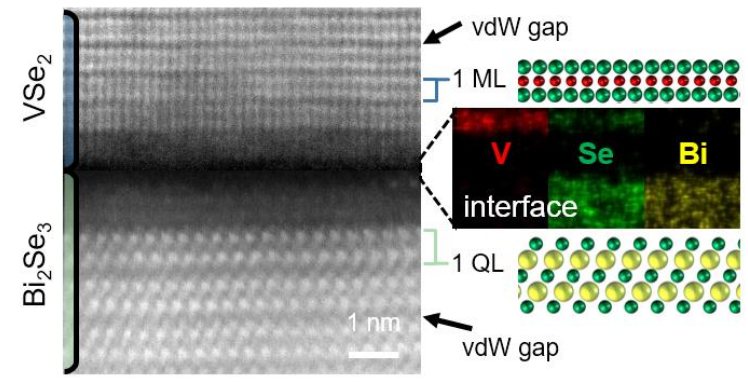

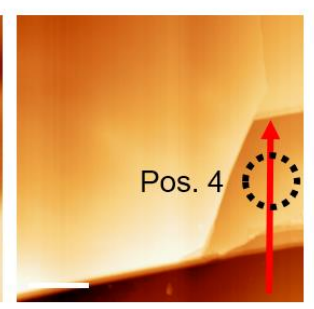

e

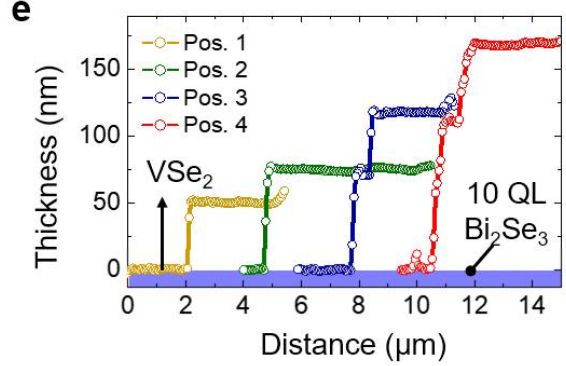

Fig. 1 Structural information and morphology of VBHs. (a) Optical image of VBHs. The regions of interest are marked as positions 1-4. The black solid line marks the boundaries of the $\mathrm{VSe}_{2}$ flake and the black dashed line indicates the $\mathrm{VSe}_{2}$ region of interest for this study. (b) Raman spectra of $\mathrm{VSe}_{2}$ and $\mathrm{Bi}_{2} \mathrm{Se}_{3}$. Inset, enlarged Raman spectra of $\mathrm{VSe}_{2}$. Each Raman active mode is indicated. (c) Cross-sectional HAADF-STEM image of a VBH. The interface between $\mathrm{VSe}_{2}$ and $\mathrm{Bi}_{2} \mathrm{Se}_{3}$ shows the empty space of the carbon protective layer deposited before slice cutting by a focused ion beam. The clear vdW gap and atomic configuration are shown. Right panel, corresponding energy-dispersive X-ray spectroscopy (EDS) elemental intensity maps for V, Se, and $\mathrm{Bi}$ along with a related schematic of the atomic structure. Note that the data of cross-sectional HAADF-STEM and EDS, which are required for deposition of carbon protecting layer, were collected after completing pump-probe experiments. (d) AFM topography images of VBHs at positions 1-4. The dashed circles indicate the spots of interest in the pump-probe measurements. 
(e) Height profiles along with the arrows at positions 1-4. Note that the measured height is the thickness of $\mathrm{VSe}_{2}$ on $\mathrm{Bi}_{2} \mathrm{Se}_{3}$. 

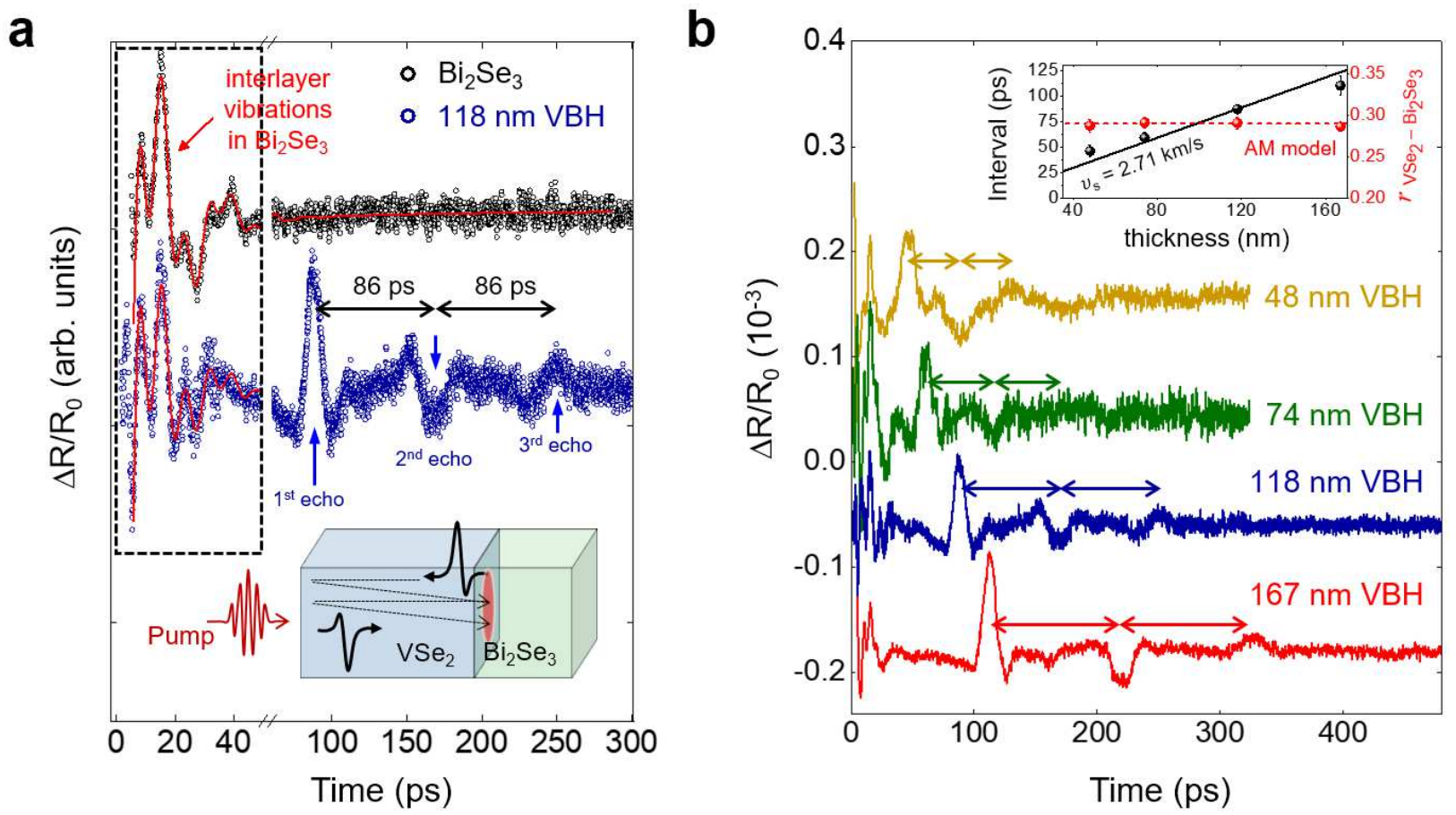

Fig. 2 Thickness-dependent CAP dynamics of VBHs. (a) Pump-probe signals of $\mathrm{VSe}_{2}-$ thickness-dependent background-free CAPs of VBHs with $1 \mathrm{eV}$ probe energy. The dashed box indicates the CAP signals from $10 \mathrm{QL} \mathrm{Bi}_{2} \mathrm{Se}_{3}$. The upward/downward arrows indicate acoustic pulse echoes at the $\mathrm{VSe}_{2} / \mathrm{Bi}_{2} \mathrm{Se}_{3}$ interfaces. Simplified schematic of pump-probe experiments and Dynamic illustration of acoustic waves in $\mathrm{VSe}_{2}$ generated by optical pump pulses and reciprocated by reflection at the air and $\mathrm{Bi}_{2} \mathrm{Se}_{3}$ interfaces (Inset). The red curves indicate fitting results with damped oscillation of interlayer vibrations in $\mathrm{Bi}_{2} \mathrm{Se}_{3}$. The time intervals between echoes ( $\left.86 \mathrm{ps}\right)$ are listed. (b) VSe 2 -thickness dependent interfacial CAP dynamics of VBHs. Obtained echo interval (black dots) versus $\mathrm{VSe}_{2}$ thickness and amplitude reflection coefficient (red dots) (Inset). The line fit (black line) to the interval data provides the sound speed of $\mathrm{VSe}_{2}(2.71 \mathrm{~km} / \mathrm{s})$. Inset: The dash red line indicates the predicted reflection coefficient based on the acoustic mismatch model for the atomically flat interface. 
a
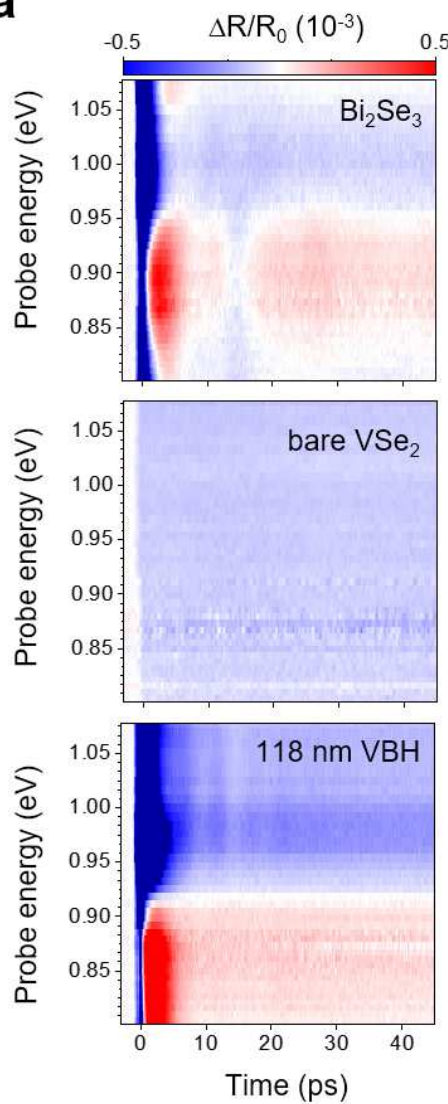

b
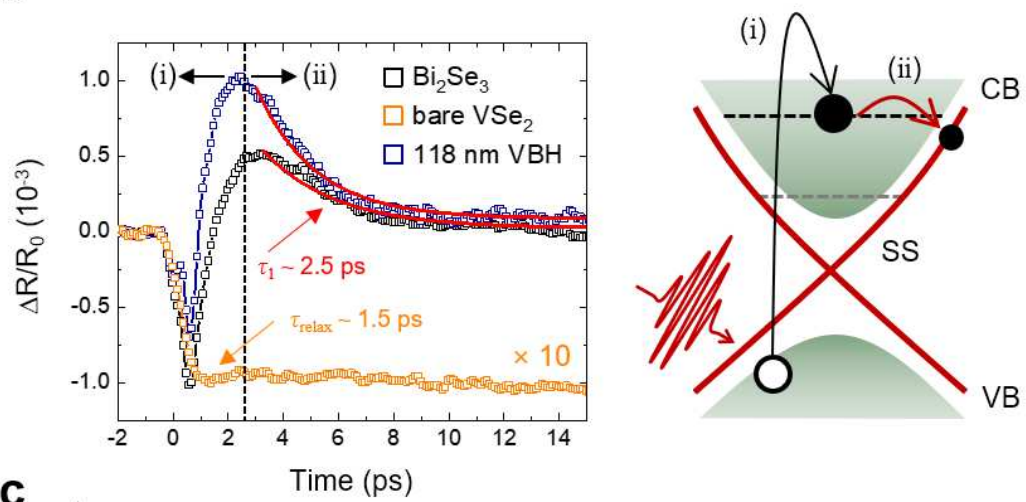

C

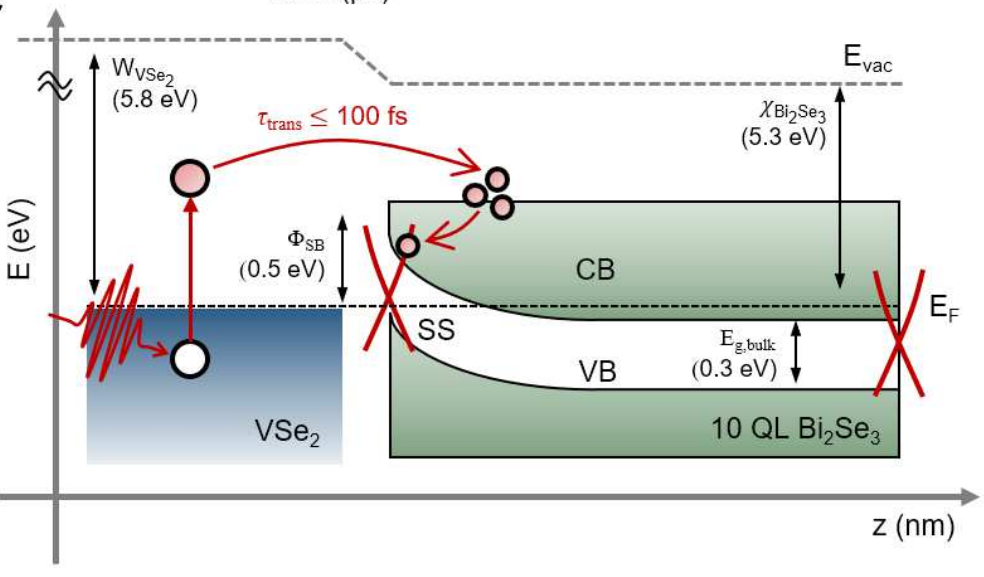

Fig. 3 (a) 2D plots of the TR spectra of $10 \mathrm{QL} \mathrm{Bi}_{2} \mathrm{Se}_{3}$, bare $\mathrm{VSe}_{2}$ film, and $118 \mathrm{~nm} \mathrm{VBHs.} \mathrm{(b)} \mathrm{TR}$ traces at each sample around $0.9 \mathrm{eV}$ probe energy. The thermalization time of bare $\mathrm{VSe}_{2}\left(\tau_{\text {relax }}\right)$ and decay time of phonon-assisted bulk-surface scattering ( $\tau_{1}$, red curves $)$ are listed. The stages of (i) and (ii) indicate the processes of ultrafast carrier dynamics in $\mathrm{Bi}_{2} \mathrm{Se}_{3}$ as illustrated in the right panel. (c) Schematic of band alignment and hot electron transfer dynamics in VBHs and. Each characteristic energy of the work function $(W)$ of $\mathrm{VSe}_{2}$, electron affinity $(\chi)$, and bandgap $\left(E_{\mathrm{g}}\right)$ of $\mathrm{Bi}_{2} \mathrm{Se}_{3}$ is shown according to previous studies. ${ }^{[11,14,62]}$ 

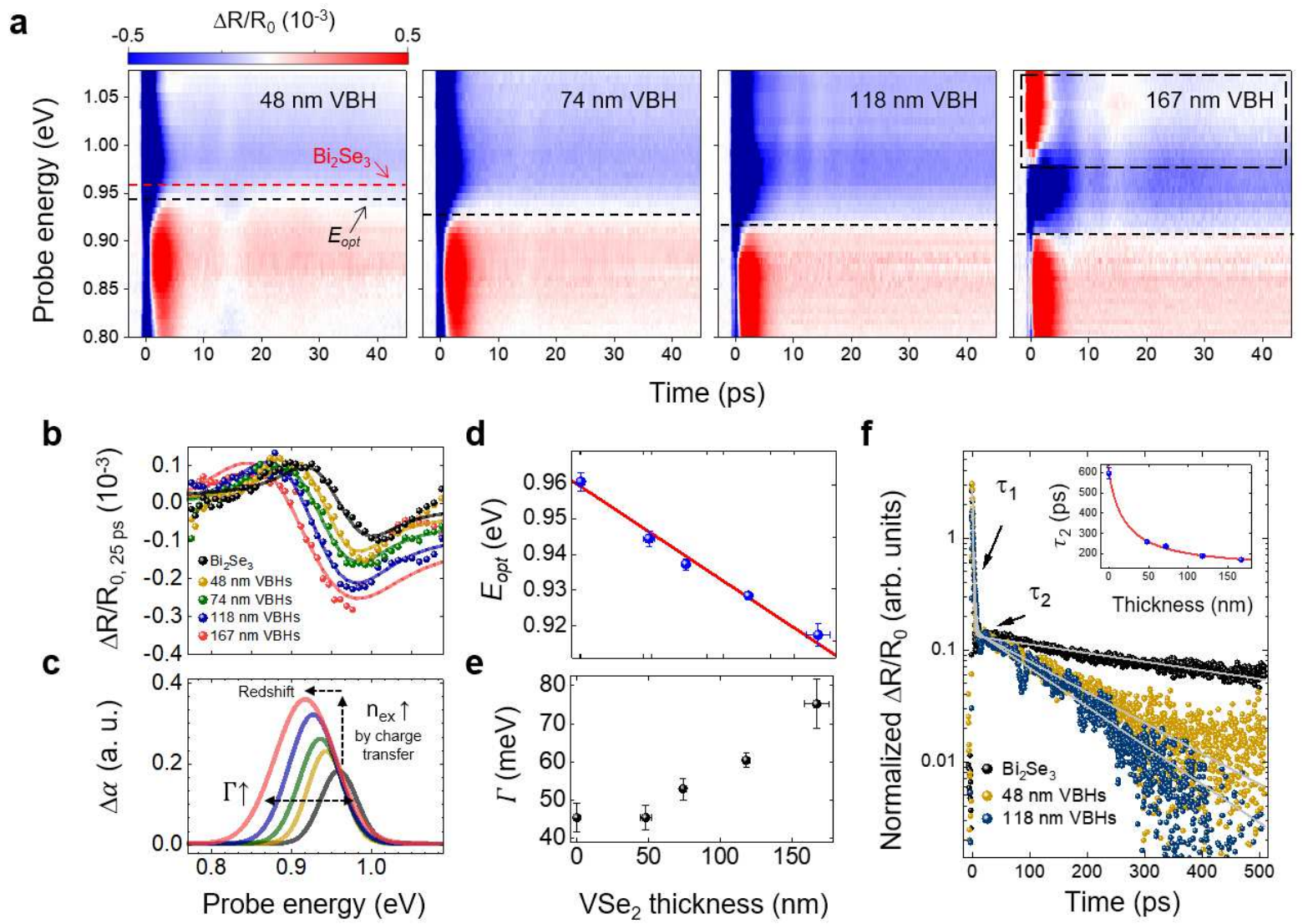

Fig. 4 (a) 2D plots of the TR spectra of VBHs. The dashed horizontal lines indicate the optical transition energy. The horizontal red dashed line indicates the optical transition energy of $10 \mathrm{QL}$ $\mathrm{Bi}_{2} \mathrm{Se}_{3}$ as shown in Figure 3a. (b) Selected TR spectral features at 25 ps with fitting curves and (c) related optical transition energy and broadening of photoinduced absorption changes. (d) $\mathrm{VSe}_{2}$ thickness-dependent optical transition energy obtained from (b, c). The line fit delivers a slope of $0.26 \mathrm{meV} / \mathrm{nm}$. (e) $\mathrm{VSe}_{2}$ thickness versus broadening factor of the optical transition estimated by model fitting. Ultrafast carrier dynamics in VBHs. (f) Dynamic evolution of TR signals in $\mathrm{Bi}_{2} \mathrm{Se}_{3}$ and VBHs. The grey fit curves indicate biexponential decays in $\mathrm{Bi}_{2} \mathrm{Se}_{3}$ and $\mathrm{VBHs}$ with $48 \mathrm{~nm}$ and $118 \mathrm{~nm} \mathrm{VBH}$. Inset: $\mathrm{VSe}_{2}$-thickness-dependent interband recombination time of metastable carrier in $\mathrm{Bi}_{2} \mathrm{Se}_{3}\left(\tau_{2}\right)$. 

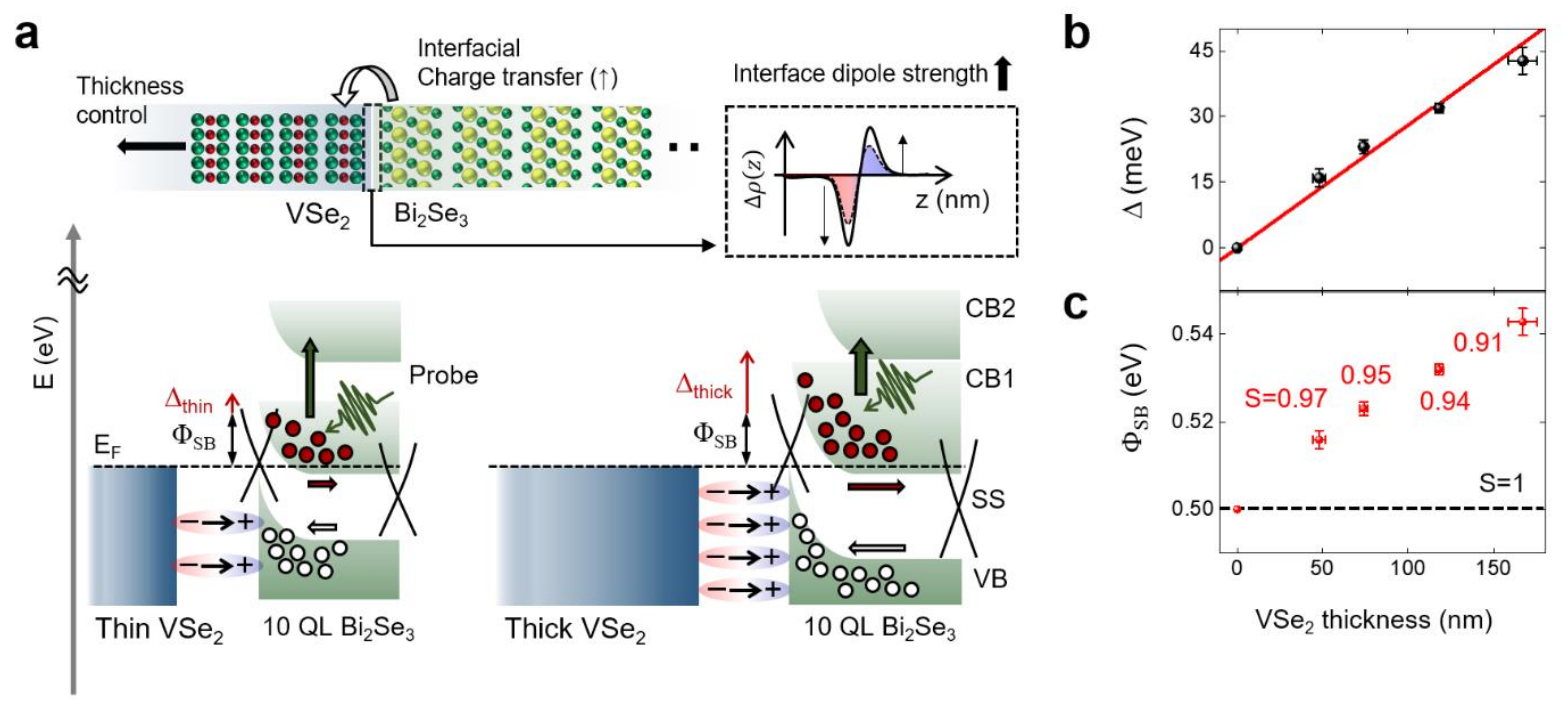

Fig. 5 Schematic of changes of the VSe 2 thickness-dependent optical transition behavior in VBHs after contact with $\mathrm{VSe}_{2}$. (a) Schematic of charge transfer and redistribution due to the thermal equilibrium by the junction at $\mathrm{VSe}_{2} / \mathrm{Bi}_{2} \mathrm{Se}_{3}$ and corresponding charge density near the junction interface. The increase of $\mathrm{VSe}_{2}$ thickness requires a larger amount of electron from $\mathrm{Bi}_{2} \mathrm{Se}_{3}$ for Fermi level alignment, leading to an increase of the interfacial dipole strength. Bottom panel: Band diagram for $\mathrm{VBH}$ with thin and thick $\mathrm{VSe}_{2}$. The electron $\mathrm{SBH}$ and band offset are illustrated by black and red vertical arrows. The green vertical arrow indicates optical transitions corresponding to probe energies. (b) $\mathrm{VSe}_{2}$ thickness-dependent band offset $(\Delta)$ by dipole interactions and (c) estimated electron $\mathrm{SBH}\left(\Phi_{\mathrm{SB}}\right)$. The expected pinning factor $\mathrm{S}$ is listed. 


\section{Supplementary Files}

This is a list of supplementary files associated with this preprint. Click to download.

- 20211227VBHCommun.Phys.SIfinal.docx 